\title{
Combined deletion of Fxr and Shp in mice induces Cyp17a1 and results in juvenile onset cholestasis
}

\author{
Sayeepriyadarshini Anakk, ${ }^{1}$ Mitsuhiro Watanabe, ${ }^{2}$ Scott A. Ochsner, ${ }^{1}$ \\ Neil J. McKenna, ${ }^{1}$ Milton J. Finegold, ${ }^{3}$ and David D. Moore ${ }^{1}$
}

\begin{abstract}
${ }^{1}$ Department of Molecular and Cellular Biology, Baylor College of Medicine, Houston, Texas, USA. ${ }^{2}$ Department of Internal Medicine, School of Medicine, Keio University, Shinjuku-ku, Tokyo, Japan. ${ }^{3}$ Department of Pathology, Baylor College of Medicine, Houston, Texas, USA.
\end{abstract}

\begin{abstract}
Bile acid homeostasis is tightly regulated via a feedback loop operated by the nuclear receptors farnesoid $\mathrm{X}$ receptor (FXR) and small heterodimer partner (SHP). Contrary to current models, which place FXR upstream of SHP in a linear regulatory pathway, here we show that the phenotypic consequences in mice of the combined loss of both receptors are much more severe than the relatively modest impact of the loss of either Fxr or Shp alone. $\mathrm{Fxr}^{-/-} \mathrm{Shp}^{-/-}$mice exhibited cholestasis and liver injury as early as 3 weeks of age, and this was linked to the dysregulation of bile acid homeostatic genes, particularly cytochrome P450, family 7 , subfamily a, polypeptide 1 (Cyp7a1). In addition, double-knockout mice showed misregulation of genes in the C21 steroid biosynthesis pathway, with strong induction of cytochrome P450, family 17, subfamily a, polypeptide 1 (Cyp17a1), resulting in elevated serum levels of its enzymatic product 17-hydroxyprogesterone (17-OHP). Treatment of WT mice with 17-OHP was sufficient to induce liver injury that reproduced many of the histopathological features observed in the double-knockout mice. Therefore, our data indicate a pathologic role for increased production of 17-hydroxy steroid metabolites in liver injury and suggest that $\mathrm{Fxr}^{-/-} \mathrm{Shp}^{-/-}$mice could provide a model for juvenile onset cholestasis.
\end{abstract}

\section{Introduction}

Originally considered endogenous surfactants required for digestion of fats and fat soluble vitamins (1), bile acids (BAs) have outgrown this simple characterization and are now implicated in multiple biological processes. For instance, BAs exhibit hormone-like properties to regulate liver regeneration $(2,3)$ and energy expenditure (4) as well as triglyceride (5) and glucose homeostasis (6). The physiological concentration of BAs is tightly regulated through a multistep feedback loop (7), involving the nuclear receptors farnesoid X receptor (FXR, also known as NR1H4) (8-10) and small heterodimer partner (SHP, also known as NR0B2) (7, 11-13), culminating in the repression of cytochrome P450, family 7 , subfamily a, polypeptide 1 (CYP7A1) (7), an enzyme that catalyzes the rate-limiting step in BA synthesis $(14,15)$. Cholestasis causes hepatic BA accumulation. Among the several mechanisms for BA retention are aberrant $B A$ synthesis and transport (16-19). Importantly, most of the BA synthesis and transport machinery (3, 7, 20-24) falls under the FXR and SHP axis of regulation.

The interplay among BAs, FXR, and SHP is complex. BAs act as endogenous ligands for FXR to autoregulate their synthesis (7), hydrophobicity (10), and transport $(21,25,26)$. FXR can also regulate BA levels via a pathway involving FGF15 and its receptor FGFR4 $(27,28)$. Moreover, $F x r$-knockout $\left(\mathrm{Fxr}^{\prime-}\right)$ mice challenged with cholic acid overload have elevated serum cholesterol and triglyceride levels $(10,29)$. SHP, an inductive target of FXR, is an atypical nuclear receptor (30) that lacks a DNA binding domain. SHP functions as a corepressor and inhibits transactivation of specific nuclear receptor partners, particularly LRH-1 $(31,32)$. While liver-specific overexpression of SHP results in depletion of the BA

Conflict of interest: The authors have declared that no conflict of interest exists. Citation for this article: J Clin Invest. 2011;121(1):86-95. doi:10.1172/JCI42846. pool (33), Shp-knockout ( $S h p^{-/-}$) mice are resistant to liver damage induced by chronic elevation of cholesterol as well as BAs $(5,34)$.

Current understanding suggests FXR functions upstream of SHP in a linear pathway $(7,24,35)$. If FXR is exclusively epistatic to SHP in a linear regulatory pathway, then $\mathrm{Fxr}^{-/-} \mathrm{Sh} \mathrm{p}^{-/-}$doubleknockout (DKO) mice should phenocopy Fxr/- mice. In contrast, we find that DKO mice display severe liver injury and biliary dysfunction as early as 3 weeks of age, and their liver size doubles within 12 weeks of age when compared with that of the individual knockouts. The biliary phenotype is associated with substantially higher Cyp7a1 expression in DKO mice relative to the single knockouts. These results strikingly differ from the modest phenotype of individual $\mathrm{Fxr}^{--}$or Shp $\mathrm{S}^{-/}$animals and indicate that FXR and SHP act coordinately to maintain biliary homeostasis.

In addition to the changes in cholesterol and BA metabolism, microarray analysis of the DKO liver identified specific alterations in the expression of genes involved in C21 steroid metabolism, with marked induction of cytochrome P450, family 17 , subfamily a, polypeptide 1 (Cyp17a1) and decreased expression of 3- $\beta$-hydroxysteroid dehydrogenase $5(H s d 3 b 5)$ and steroid 5- $\alpha$-reductase $1(\operatorname{Srd5a1})$. The induction of Cyp17a1 correlated with significantly elevated serum levels of one of its primary products, 17-hydroxyprogesterone (17-OHP). Liver toxicities have been associated with synthetic 17- $\alpha$ hydroxysteroids, including 17- $\alpha$ ethinyl estradiol and 17- $\alpha$ alkylated (17aa) androgens (36-38). To test its potential contribution to DKO liver injury, we challenged WT mice with 17-OHP for 5 days. 17-OHP treatment was sufficient to induce liver injury, producing histological changes similar to those observed in DKO mice, including microsteatosis, focal inflammation, and ballooning degeneration. Overall, these results identify the DKO mice as a model of juvenile onset cholestasis and suggest an unexpected contribution of endogenous $17-\mathrm{OHP}$ and potentially other Cyp17a1 products in hepatotoxicity. 
A

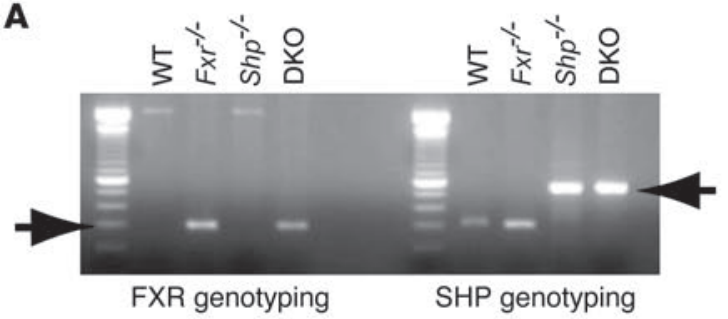

B

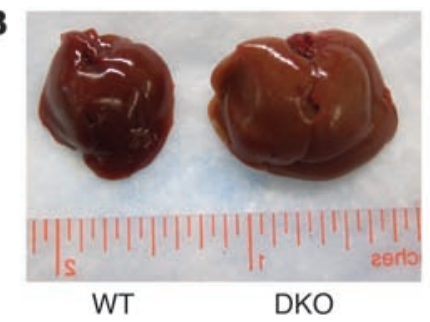

C

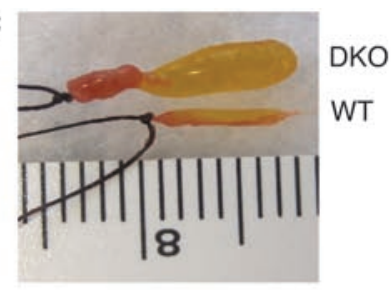

D

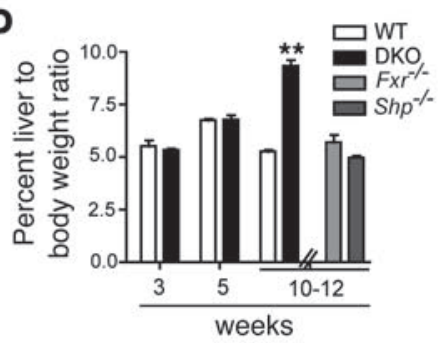

$\mathbf{E}$

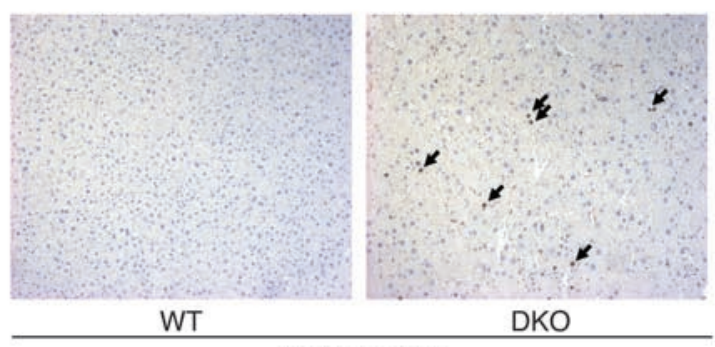

Ki-67 staining

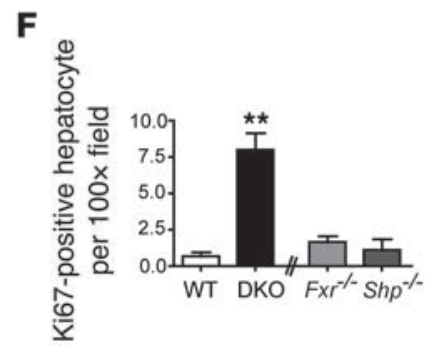

Figure 1

Increased liver and gallbladder size indicates hepatobiliary dysfunction in FXR/SHP DKO mice. (A) Genotyping PCR confirms the generation of DKO mice. (B) DKO mice exhibit hepatomegaly. Increased (C) gallbladder and (D) liver size occurs only in 10- to 12-week-old DKO mice. (E) Increased hepatocyte Ki-67 staining, marked by arrows, indicates increased cellular proliferation in the 10-week-old DKO liver. Brown-stained nuclei show Ki-67-positive cells, and its quantification is shown in F. Original magnification, $\times 125(\mathbf{E})$. Data are presented as mean \pm SEM, $n=8-15 .{ }^{* *} P<0.001$ when compared with WT.

\section{Results}

Loss of FXR and SHP results in increased size of the hepatobiliary tract in

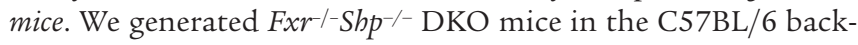
ground by breeding homozygous $\mathrm{C} 57 \mathrm{BL} / 6 \mathrm{Fxr}^{-1}$ mice with similarly congenic $S h p^{-1-}$ mice (Figure 1A). While no gross changes in liver size were visible up to 5 weeks of age, the liver-to-body weight ratio of the DKO mice doubled between 10 and 12 weeks of age in comparison with that of WT or the individual $\mathrm{Fxr}^{-1}$ or $\mathrm{Shp}^{-/-}$mice (Figure 1, B and D). A significant increase in gallbladder size and its biliary contents was also observed in the DKO mice compared with that of WT (Figure 1C) or the single $\mathrm{Fxr}^{-/}$or $\mathrm{Shp}^{-/-}$mice (data not shown). To determine whether the hepatomegaly in DKO mice results from cellular proliferation or hypertrophy, we performed Ki-67 immunostaining on liver sections from WT and DKO mice to identify DNA synthesis as a measure of cellular proliferation. A 10-fold increase in nuclear Ki-67 staining in hepatocytes of DKO mice compared with that of WT mice indicated that the increase in weight of the liver seen in the DKO mice is primarily due to enhanced cellular proliferation (Figure 1, E and F).

Juvenile DKO mice display severe liver injury. We next used histological, biochemical, and ultra-structural analyses to assess DKO liver structure and function. H\&E staining revealed microsteatosis, focal hepatocellular necrosis, focal lobular inflammation, proliferation of bile ducts, and increased mitoses of hepatocytes in the DKO livers as early as 5 weeks relative to WT mice (Figure 2, A and C). By 12 weeks of age, DKO mice exhibited significant hepatic lipid accumulation (Supplemental Figure 1; supplemental material available online with this article; doi:10.1172/JCI42846DS1), accompanied by cytoplasmic swelling and diffuse hepatocellular injury (Figure 2D). Electron microscopy (Figure 2, E-H) revealed progressive dilatation of mitochondrial cristae (Figure 2, G and H) and ER (data not shown). Cytokeratin 19 (CK19) immunostaining confirmed ductular proliferation in DKO mice (Figure 2, I and J).
Next, we evaluated liver injury in DKO mice by measuring serum concentrations of ALT, AST, and bilirubin. Consistent with the histological findings, DKO mice exhibited up to 20- and 6-fold increases in ALT and AST levels, respectively, indicative of significant hepatocellular injury (Figure 2, K and L). There was a striking increase in conjugated bilirubin at 12 weeks (Figure 2M), consistent with decreased bile flow. In contrast to DKO mice, $\mathrm{Fxr}^{-1-}$ mice exhibited a much lower degree of liver necrosis, and $\mathrm{Shp}^{-1-}$ mice showed no significant changes in any of the above parameters (Supplemental Figure 2).

Increased BAs cause liver injury in DKO mice. We predicted that liver injury in the DKO mice might arise from BA accumulation in the absence of a negative feedback mechanism. As expected, we observed a significant increase in both serum and hepatic BA levels in the DKO mice when compared with those in WT or in the single $\mathrm{Fxr}^{-/}$or Shp ${ }^{-/}$mice (Figure 3, A and B, and Supplemental Figure 3, A-D). This increase was evident in 3-week-old mice and strongly correlated with the increased serum ALT and AST levels. BA levels were also elevated in the biliary and intestinal compartments of DKO mice (Figure 3, C and D). Hepatic and serum levels of cholesterol, the precursor for BA production, were modestly increased in DKO mice relative to WT mice (Figure 3, E and F). Fxr ${ }^{-/}$mice also showed elevated cholesterol levels in serum but not in the liver, whereas in $S h p^{-1-}$ mice, hepatic and serum cholesterol levels were modestly elevated and decreased, respectively (Supplemental Figure 3, E and F). The increase in hepatic cholesterol levels observed in DKO and $\mathrm{Shp}^{-/-}$ mice was consistent with the induction of mRNA for HMGCoA reductase, an enzyme involved in the rate-limiting step for cholesterol synthesis, in DKO mice ( $1.52 \pm 0.2$ fold change in mRNA expression compared with WT mice; $P<0.05)$ and in $S h p^{-1-}$ mice (1.55 \pm 0.04 fold change in mRNA expression compared with WT mice; $P<0.01)$ at 12 weeks of age. 
WT

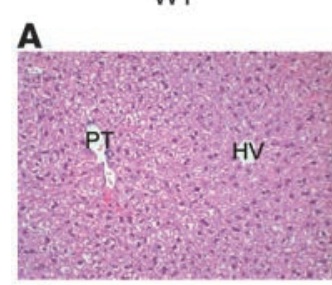

E

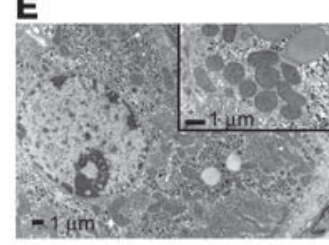

I

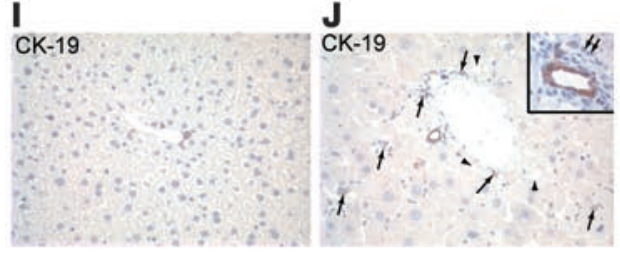

F
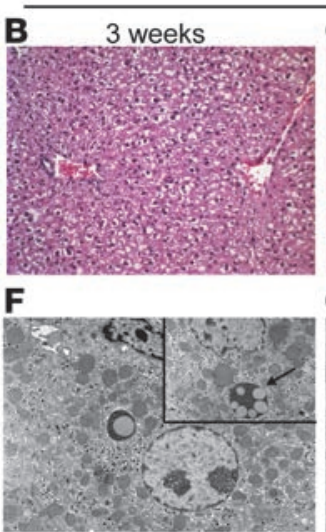

K

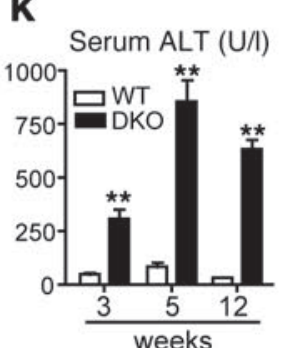

G
DKO
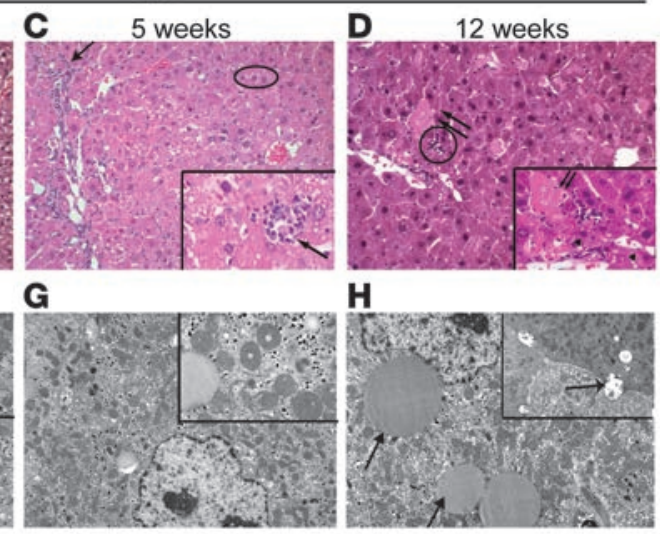

L

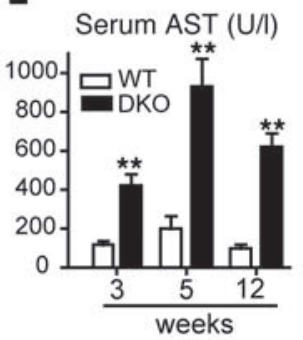

\section{M}

Serum bilirubin $(\mathrm{mg} / \mathrm{dl})$

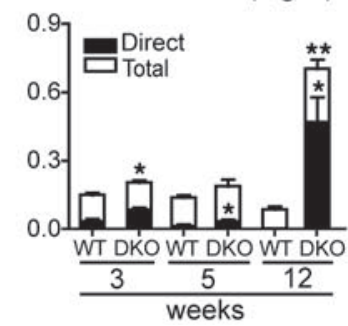

Figure 2

Severe liver injury is visible in DKO mice. (A) Histology of WT liver shows uniform hepatocytes in regular cords between portal tract (PT) and terminal hepatic vein (HV). Hepatocytes are pale because of abundant glycogen. (B) At 3 weeks, DKO hepatocytes are diffusely altered, with slight ballooning of cytoplasm and slightly increased mitoses. (C) At 5 weeks, there is proliferation of bile ducts in portal tracts (arrow) and microsteatosis (oval). Inset shows focal inflammation (arrow). (D) By 12 weeks, hepatocellular necrosis is frequent (arrows), and there is focal lobular inflammation (circle). Inset highlights necrosis at 12 weeks (arrows). (E) Electron micrographs of the corresponding samples show small lipid droplets in cytoplasm of a normal WT hepatocyte. Inset shows normal mitochondria. (F) At 3 weeks, many hepatocyte mitochondria are enlarged with a dense matrix and focally dilated cristae (inset, arrow). (G) At 5 weeks, a hepatocyte nucleus displays wrinkling of the membrane, indicating early necrosis, and more mitochondria have frequent dilatation of cristae (inset). (H) At 12 weeks, considerable steatosis with larger droplets is evident in the cytoplasm of many hepatocytes, and there is focal dilatation of bile canaliculi with loss of microvilli (inset, arrows). (I and $\mathbf{J}$ ) CK19 staining of WT and DKO mice. The DKO liver shows ductular proliferation (arrows) and lipid droplets (arrowheads). Inset shows lymphocytes surrounding proliferating ducts. Original magnification: $\times 100$ (A-D); $\times 400$ (insets, C and D); $\times 1,500$ (E-H); $\times 3,000$ (insets, E-H) $\times 360$ (I and J); $\times 400$ (inset, J). Increased serum (K) ALT, (L) AST, and (M) bilirubin levels as early as 3 weeks after birth confirm liver dysfunction. Data are presented as mean \pm SEM, $n=8-10 .{ }^{*} P<0.05,{ }^{* \star} P<0.001$ when compared with WT.

Because we observed an absolute increase in BA levels, we determined their specific composition using HPLC analysis. Fxr ${ }^{-1-}$ mice showed a net increase in hydrophobic BAs in serum, while Shp $p^{-/-}$ mice showed a net increase in the liver (Supplemental Figure 3, $\mathrm{G}-\mathrm{I})$. However, the DKO mice had elevated hydrophobic BA in both serum and liver (Figure 3, G-I).

Disrupted BA transport and synthesis causes cholestasis in DKO mice. To decipher the biochemical basis for the dramatic increase in BA levels in the DKO livers, we performed a systematic and quantitative analysis of the hepatic expression of a panel of genes involved in BA synthesis, transport, and elimination using NanoString assays (39). While DKO mice exhibit strong increases relative to WT mice in genes involved in classical BA synthesis (e.g., Cyp7a1), the expression of genes involved in acidic BA synthesis (e.g., Cyp27a1) was significantly lowered when compared with that of WT mice (Figure 4, A and B). We also found a significant increase in the expression levels of Cyp8b1 (Figure 4D), which encodes sterol $12-\alpha$ hydroxylase, an enzyme that directs BA precursors toward hydrophobic BAs. The induction of both Cyp7a1 and Cyp8b1 genes is consistent with the net increase in both total and hydrophobic BAs seen in DKO mice. Furthermore, the livers of DKO mice exhibited aberrant regulation of genes encoding proteins that transport BA into the bile, such as $A b c b 11, A b c b 1 a$, and $A b c c 2$ (Figure $4, E-G)$. In particular, the dramatic reduction in transcript levels of Abcb11, a key BA efflux gene, supports the accumulation of hepatic BA observed in DKO animals. Abcb11 repression was less in $\mathrm{Fxr}^{-1}$ mice, particularly at 12 weeks, and was absent in $\mathrm{Shp}^{-/-}$mice (Supplemental Figure 4B). $A b c c 3$ and $A b c c 4$, which encode transporters responsible for BA efflux into the systemic circulation, were strikingly induced (10 and 30 fold, respectively), an observation consistent with the increased serum BA levels observed in the DKO mice (Figure 4, I and J). Similarly, genes encoding proteins responsible for BA uptake into hepatocytes were significantly downregulated (Figure 4, K and L). As an apparent compensatory mechanism to decrease the overall BA load, genes encoding phase I and phase II enzymes in BA clearance (Figure 4, M-P) were induced in DKO mice.

$\mathrm{Fxr}^{-1}$ and $S h p^{-1-}$ single mutants showed moderate increases in Cyp7a1 and Cyp8b1 gene expression but little to no change in the alternate BA synthesis pathway (Supplemental Figure 4, A-D). At the protein level, Cyp7a1 was increased by 11.6 fold in the DKO mice, compared with the 4-fold and 1.2-fold induction in $\mathrm{Fxr}^{-1-}$ and 
A

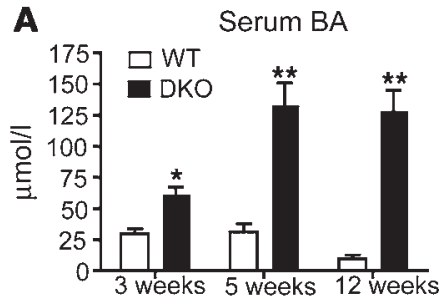

E

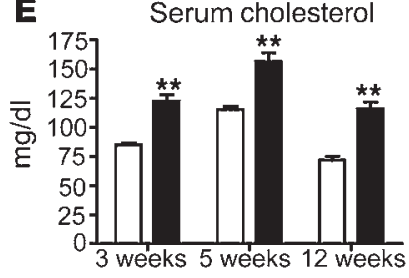

G

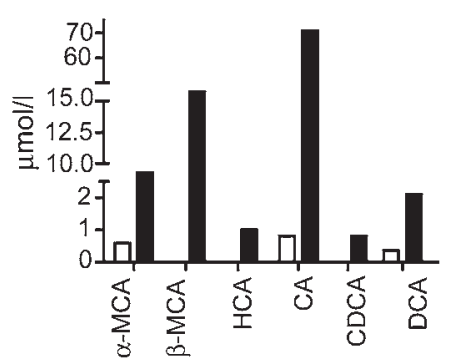

B

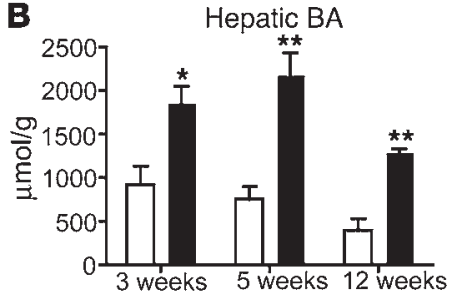

F

Hepatic cholesterol
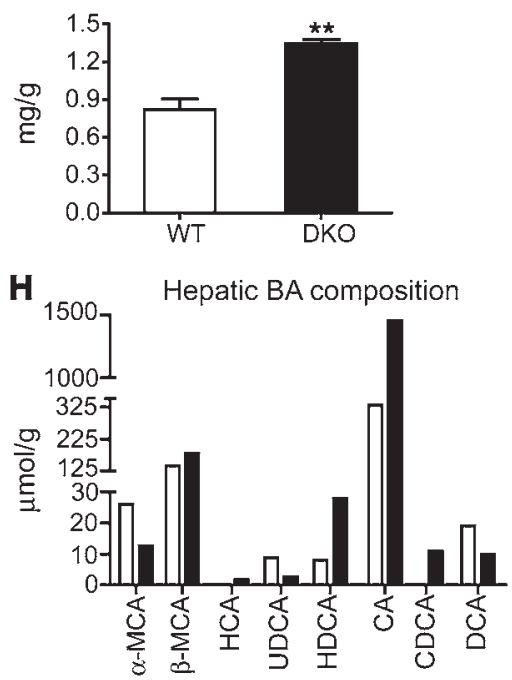

C Biliary BA
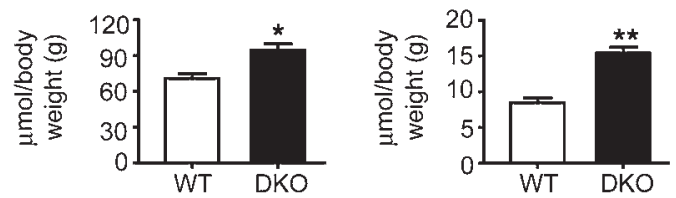

Figure 3

DKO mice exhibit juvenile cholestasis. BA accumulation in (A) serum and (B) liver is observed as early as 3 weeks only in the DKO mice. (C and D) Biliary and intestinal BA are increased in DKO mice. (E and F) Cholesterol levels, a precursor for BA, are also higher in DKO mice but not in WT mice. (G-I) BA composition reveals increased hydrophobic pool size in DKO mice. $\alpha$-MCA, $\alpha$-muricholic acid; $\beta$-MCA, $\beta$-muricholic acid; HCA, hyocholic acid; CA, cholic acid; CDCA, chenodeoxy cholic acid; DCA, deoxy cholic acid; UDCA, ursodeoxy cholic acid; HDCA, hyodeoxy cholic acid. Data are presented as mean $\pm \mathrm{SEM}, n=6 .{ }^{*} P<0.05,{ }^{* *} P<0.001$ when compared with WT.

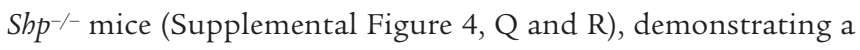
coordinate role for FXR and SHP in Cyp7a1 regulation and largely accounting for the significantly higher BA levels in the DKO mice compared with those in the individual knockout mice (Figure 4, Q and $\mathrm{R}$ ). The expression levels of most genes regulating BA transport and elimination in $\mathrm{Fxr}^{-/}$(10) and $\mathrm{Shp}^{-/-}$(11) mice exhibited changes syndirectional to those in the DKO mice, albeit lower in amplitude (Supplemental Figure 4, E-P). Taken together these data explain for the overall increase in BA levels observed in the DKO mice.

Hepatic gene signature of DKO mice. To further explore the basis for the increased hepatopathology in DKO mice, we used microarrays to profile gene expression in the livers of 3- and 5-week-old WT, $\mathrm{Fxr}^{--}, \mathrm{Shp}^{-/-}$, and DKO mice. The gene expression analysis was carried out at a relatively early age in order to minimize secondary gene expression changes, pursuant to the severe liver injury in the DKO animals. At 5 weeks of age, we believe that at least $50 \%$ of the observed differential gene expression in $\mathrm{DKO}, \mathrm{Fxr}^{--}$, and $\mathrm{Shp}^{-/}$ mice relative to WT mice (Figure 5A) was unique to their respective genotypes. Interestingly, the DKO mice showed a 39\% probe set overlap with the $\mathrm{Fxr}^{-/}$mice and only 5\% probe set overlap with the $S h p^{-1-}$ mice, indicating that the loss of FXR contributes more to biliary dysfunction than does the loss of SHP in the DKO animals.

Gene Ontology (GO) overrepresentation analysis $(40,41)$ of the differentially expressed genes specifically regulated by FXR and SHP (Supplemental Excel file 1) or unique to DKO mice (Figure 5B) iden- tified a number of what we believe to be new functional targets. Those altered specifically in the DKO livers include gene networks regulating steroid, BA, and cholesterol metabolism. In particular, Cyp17a1, an important enzyme in both the biosyntheses of pregnenolone and hydroxy progesterone via the C21 steroid pathway (42-44) was dramatically induced up to 20 fold in the DKO livers (Figure 5D). In contrast, transcript levels of $H s d 3 b 5$ were negligible at 3 weeks (Figure $5 \mathrm{E}$ ), while the expression of $\operatorname{Srd5} 1 \mathrm{1}$ (Figure $5 \mathrm{~F}$ ) was reduced to $50 \%$.

17-OHP causes liver injury. Cyp $17 \mathrm{a} 1$ is a single enzyme but has both $17 \alpha$-hydroxylase and 17,20-lyase activities. It first catalyzes the 17-hydroxylation of progesterone or pregnenolone, which may then be converted to DHEA or androstenedione, respectively, via its lyase activity. The induction of Cyp17a1 in DKO mice at the mRNA and protein levels (Figure 6, A and B) resulted in a significant increase in circulating 17-OHP levels but not in DHEA levels relative to those of WT mice (Figure 6, C and D). 17-OHP is normally synthesized in the adrenal and the gonads and is considered nontoxic (45). However, liver toxicities have been reported with synthetic 17- $\alpha$ hydroxylated steroids, including 17- $\alpha$ ethinyl estradiol, and 17aa androgens. Interestingly, short-term treatment of WT mice with 17-OHP was sufficient to cause liver injury, as demonstrated by increased serum ALT, AST, and bilirubin levels. This was accompanied by a 26 percent increase in liver-to-body weight ratio (Figure 6, E-G), with no significant effect on serum and hepatic BA levels (Figure 6, $\mathrm{J}$ and $\mathrm{K})$. Histological analysis demonstrated focal inflammation, 
BA synthesis

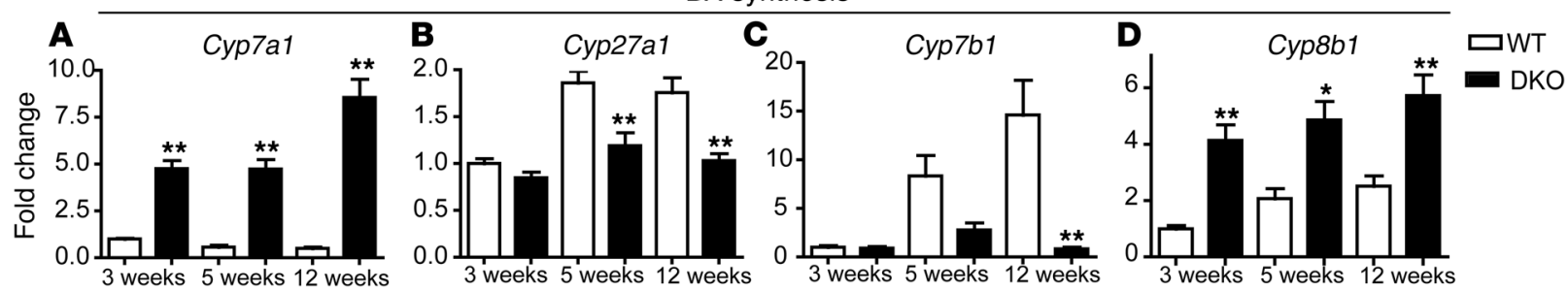

BA efflux into bile

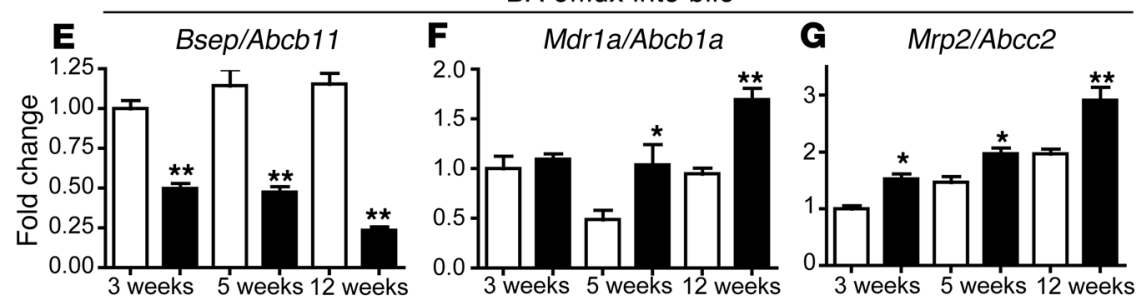

$B A$ efflux into circulation

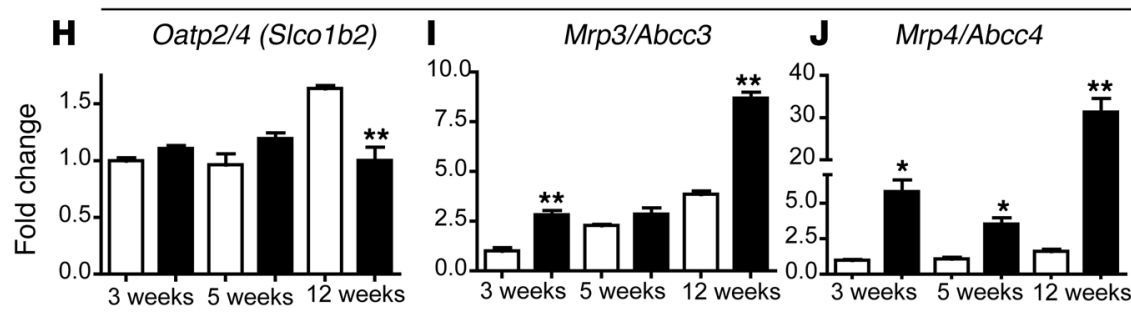

BA uptake into hepatocytes

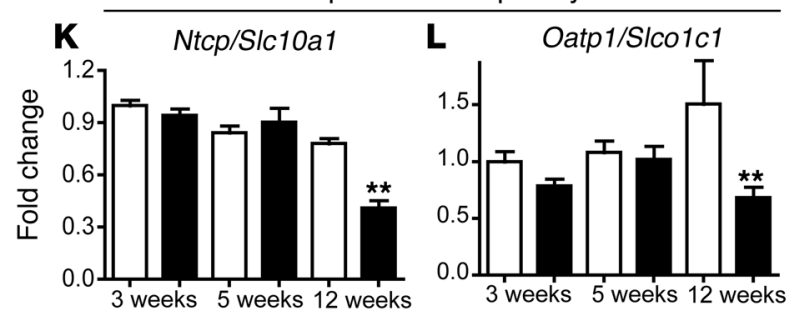

Phase I metabolism

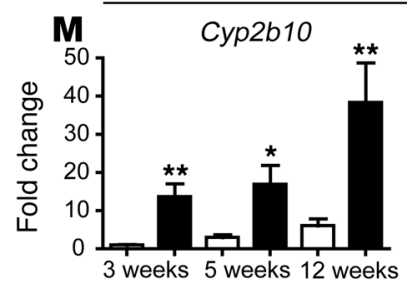

N Cyp3a11
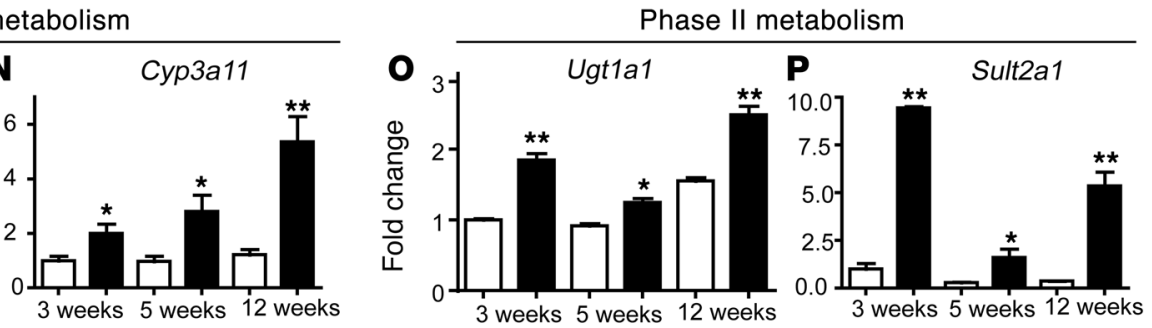

Q

WT

DKO

CYP7A

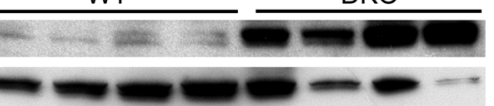

$\beta$-Actin

\section{R Cyp7A1 protein}

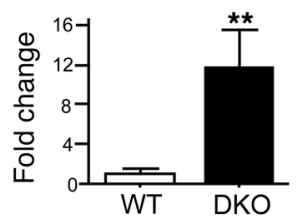

Figure 4

Dysregulation in BA synthesis and transport pathways results in severe cholestasis in DKO mice. Total RNA $(n=4)$ for each time point was prepared, and gene expression was analyzed in duplicates using NanoString technology. Genes involved in synthesis of (A) neutral and (D) hydrophobic BA are increased but those involved in acidic BA (B and $\mathbf{C})$ are decreased. (E-G) Aberrant expression of genes involved in BA efflux into bile. $(\mathbf{H}-\mathbf{J})$ Genes involved in BA efflux into circulation are increased, whereas $(\mathbf{K}$ and $\mathbf{L})$ those involved in BA uptake into liver are decreased. (M-P) Genes involved in BA detoxification are increased. (Q) Cyp7A protein levels are induced dramatically in DKO animals and are quantified in $\mathbf{R}$. Throughout the figure, fold change is shown with respect to WT at 3 weeks. Data are presented as mean \pm SEM. ${ }^{\star} P<0.05$, ${ }^{* *} P<0.001$ when compared with their respective age-matched WT animals. 
A
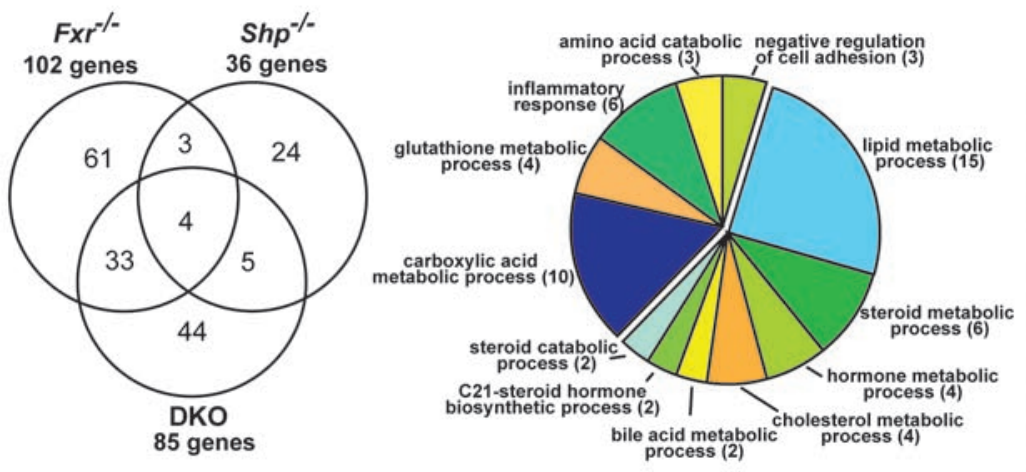

D
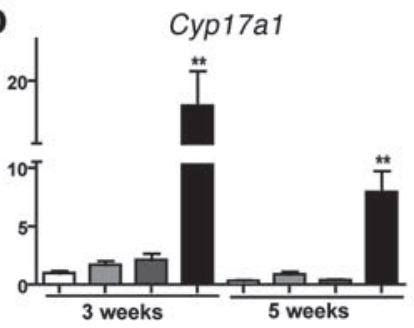

E

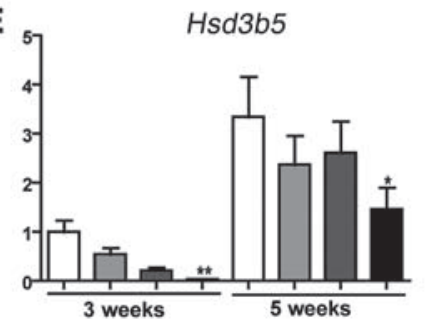

C
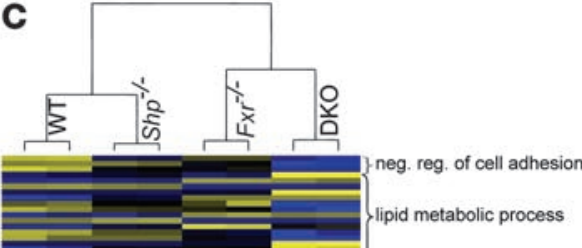

lipid metabolic process steroid metabolic process glutathione metabolic process hormone metabolic process steroid catabolic process cholesterol metabolic process inflammatory response C21-steroid hor. biosyn. process carboxylic acid metabolic process amino acid catabolic process

Figure 5

Loss of FXR and SHP affects steroid and lipid metabolism. RNA from liver of 5-week-old mice $(n=6)$ was analyzed by microarray. (A) Venn diagram comparing $\mathrm{Fxr}^{-1-}$ and Shp ${ }^{-1-}$ mice versus DKO mice. The selection criteria used was fold change $>2.3$ and $P<0.0001$. (B) Classification of a subset of target genes, which are uniquely altered in DKO mice. The numbers in parentheses indicate the number of genes in that category. (C) Relative expression of 63 probe sets for genes from the GO analysis. Each knockout is represented by data from 2 samples, and each samples is a pool of $n=3$ mice. DKO gene signature, which we believe to be novel, was validated by NanoString analysis $(n=4)$. neg., negative; reg., regulation; hor., hormone; biosyn., biosynthetic. (D-F) Expression of C21 steroid metabolic genes. Data are presented as mean \pm SEM, $n=4$ mice per group. ${ }^{*} P<0.05,{ }^{\star *} P<0.001$ when compared with their respective age-matched WT.

periportal cholangitis, ballooning degeneration, and microsteatosis (Figure 6, $\mathrm{L}$ and $\mathrm{M}$ ) in these livers, similar to the DKO mice. The dose chosen $(125 \mathrm{mg} / \mathrm{kg})$ is lower than that used to delay preterm labor in human patients $(250 \mathrm{mg} / \mathrm{kg})$. This resulted in 10 - and 5 -fold higher serum 17-OHP levels in comparison with WT vehicle and naive DKO mice, respectively, which is less than the levels that can be reached in congenital adrenal hyperplasia (46). From these results, we conclude that high levels of 17-OHP, a product synthesized by Cyp17a1, are toxic to the liver, and its local production may contribute to the severe pathology observed in the DKO mice.

\section{Discussion}

Cholestasis is a term encompassing diseases in which bile flow is reduced or arrested and substances normally excreted into bile are retained. Hypercholemia, or increased serum BA concentration, is used as a universal indicator of cholestasis, which may or may not be accompanied by jaundice and elevated serum bilirubin. There are numerous etiologies of cholestasis, including genetic diseases such as progressive familial intrahepatic cholestasis (PFIC1PFIC3), BA synthesis defects, $\alpha 1$ anti-trypsin deficiency, Alagille syndrome, and mitochondrial hepatopathies $(18,47)$. Like the DKO mice, the cholestasis associated with several of these genetic syndromes is both early onset and severe.

Although genetic loss of both FXR and SHP is not likely to contribute to human cholestatic syndromes, PFIC1 patients show decreased ileal FXR activity $(48,49)$, and SNPs $518 \mathrm{~T}>\mathrm{C}$ and $238 \mathrm{~T}>\mathrm{C}$ in FXR have been associated with intrahepatic cholestasis of preg- nancy (ICP) (50,51). More importantly, recent data show that both FXR and SHP expression is dramatically reduced by $90 \%$ or more in PFIC1, PFIC2, and in biliary atresia (52). The Fxr $\mathrm{Fhp}^{-/-}$DKO mouse model exhibits a range of cholestatic dysfunctions, including enhanced BA synthesis and a significant decrease in mRNA expression of $A b c b 11$, the bile salt export transporter implicated in PFIC2. These observations indicate that cholestatic syndromes with quite disparate primary causes show a number of similarities and that combined dysfunction of the FXR/SHP axis may be central to many different forms of cholestasis.

DKO mice completely lack the FXR/SHP-mediated BA synthesis negative feedback loop, leading to aberrant BA accumulation in liver and spillover of this excess to varying degrees into the serum, bile, and intestine. The DKO mouse model develops cholestasis as early as 3 weeks of age and as such will be an excellent tool to study earlyonset cholestasis. Additionally, unlike that in diet-induced cholestasis (53), the DKO mice accumulate a range of both hydrophilic and hydrophobic BAs, similar to that in most clinical settings $(54,55)$. Despite an early onset of liver damage, DKO mice remain viable for more than a year. The hepatocyte proliferation observed in this model cannot be accounted for by the FXR-dependent regenerative response (2). However, our unpublished data demonstrates that oval cells may support proliferation of hepatocytes in DKO mice (56).

Comprehensive analysis of the DKO phenotype has identified a coordinate role for FXR and SHP in biliary homeostasis, in contrast to the simple linear regulatory pathway suggested by the current models $(7,24)$. As in earlier studies $(13,34)$, the $S h p^{-1-}$ mice 

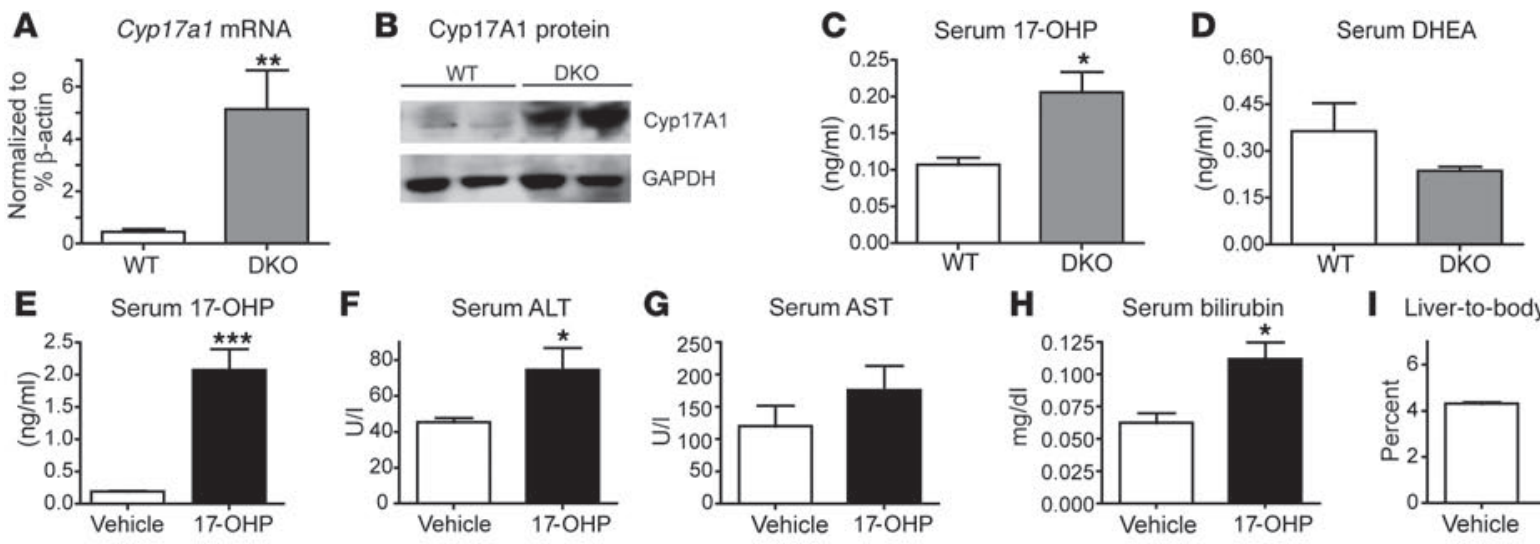

I Liver-to-body weight ratio
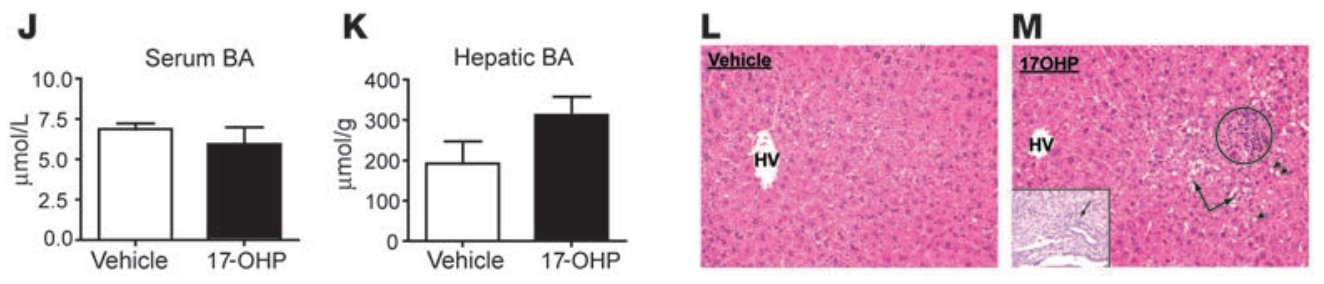

\section{Figure 6}

17-OHP contributes to the liver injury in DKO mice. Increase in (A) Cyp17a1 mRNA and (B) Cyp17a1 protein strongly correlates with the increased circulating levels of (C) 17-OHP but not (D) DHEA in DKO mice compared with the WT mice. (E-M) Ten-week-old WT mice $(n=5)$ were treated with either 17-OHP or vehicle for 5 days. (E) Circulating 17-OHP levels after the exogenous injection regimen. (F-H) 17-OHP causes liver injury and (I) increases liver size but does not change ( $\mathbf{J}$ and $\mathbf{K}$ ) serum and hepatic BA levels. (L and $\mathbf{M})$ H\&E staining shows focal inflammation (circle), lipid droplets (arrowheads), and ballooning degeneration (arrows), and the inset shows periportal cholangitis only in 17-OHP-treated mice. Original magnification, $\times 250$ (L and M); $\times 400$ (inset). Data are presented as mean \pm SEM, (A) $n=4$ mice per group; (C-K) $n=4-5$ mice per group. ${ }^{*} P<0.05$, ${ }^{* *} P<0.001$ when compared with WT; ${ }^{* *} P<0.0001$ when compared with vehicle-treated mice.

exhibited a relatively small increase in Cyp7a1 mRNA levels, with no elevation of hepatic or serum BA levels (Supplemental Figure 4). $\mathrm{Fxr}^{-1}$ mice had modest increase in Cyp7a1 expression, lesser Abcb11 repression, and a much lower increase in serum and hepatic BA levels compared with those in DKO mice (Supplemental Figure 3). However, $\mathrm{Fxr}^{-1}$ mice accumulate hepatic BAs, along with activation of detoxification pathways, at a much later age (10,57). SHP expression in the $\mathrm{Fxr}^{-/}$mice is low but not absent (10). It is obvious from the large increase of Cyp7a1 protein (Figure 4Q) in DKO mice compared with that in the individual knockouts that this residual SHP expression is sufficient to partially restrain BA overproduction and maintain relatively normal BA homeostasis in the $\mathrm{Fxr}^{-/}$liver.

BA overload is known to induce oxidative stress, hepatocyte death, and mitochondrial abnormality leading to liver damage (58). All of these insults are visible in the DKO livers, indicating BAs as the causative factor for liver injury in these mice. Interestingly, these alterations are not accompanied by bile retention, and there is only mild histological evidence for canalicular dilatation at 12 weeks. Electron microscopy points to early abnormalities in the cristae of the mitochondrion as a marker of BA toxicity $(16,58)$.

An unexpected observation from the gene array study was the robust induction of Cyp17a1 in the DKO livers. Recent literature and in silico promoter analysis of Cyp $17 \mathrm{a} 1$ indicate putative binding motifs for a variety of nuclear receptors, including FXR, VDR, HNF4 (59), LRH-1 (60), and RXR, as well as motifs for GATA6 and MED1 (61). Therefore, it is likely that induction of Cyp17a1 in the DKO liver reflects coordinated suppression by FXR and SHP under normal circumstances.
17-OHP, an enzymatic product of Cyp $17 \mathrm{a} 1$, is a naturally occurring steroid. 17-OHP neither binds nor activates the progesterone receptor (62) but is produced at high levels during pregnancy and classical congenital adrenal hyperplasia (63). We are not aware of previous reports linking it to hepatoxicity, but increased liver function tests (serum AST, ALT, GGT, and ALP) in patients with CAH (64) have been reported. There are also indications that synthetic 17-hydroxylated steroids and their metabolites have deleterious effects in the liver. 17- $\alpha$ Ethinylestradiol is a well-known inducer of cholestasis in rodent models (19) that requires ER $\alpha$ to inhibit bile flow (65). In addition, athletes and body builders who use 17 aa steroids over a period of time commonly suffer from liver injury and cholestasis (36-38). 17aa steroids undergo first-pass metabolism in the liver, resulting in oxidation at the $\mathrm{C} 2$, reduction at the $\mathrm{C} 3$, and hydroxylation at the $\mathrm{C} 17$ (66) position on the steroid ring. Interestingly, patients with liver disease show increased $17-\alpha$ hydroxylase activity (67). Furthermore, progesterone treatment to prevent preterm labor predisposes patients to onset of $\operatorname{ICP}(68,69)$. Consistent with these reports, the liver injury in the DKO mice is associated with elevated serum levels of 17-OHP. To test whether this induction is adaptive or deleterious, we treated WT mice with 17-OHP at doses comparable to those used to delay preterm labor in human patients. This increased circulating 17-OHP to a level greater than that observed in the DKO mice but less than what is observed in human congenital adrenal hyperplasia (ref. 46 and Figure 6E). This treatment induced similar histological changes to those observed in DKO mice, including focal microsteatosis, ballooning degeneration, and focal inflammation, raising the prospect that increased production of 17-hydroxy steroid metabolites may contribute to liver injury. 
Overall, we conclude that $\mathrm{Fxr}^{-/-} \mathrm{Sh} \mathrm{p}^{-/-}$DKO mice suffer from extensive liver damage associated with BA overload that is much greater than that observed in either single knockout, indicating a coordinate role for both nuclear receptors in biliary homeostasis. Based on the numerous features shared by the DKO mice and patients with severe, early-onset human cholestatic syndromes, including markedly decreased activity of both FXR and SHP (52), we suggest that they provide a unique murine model of juvenile cholestasis.

\section{Methods}

Animals. Individual $\mathrm{Fxr}^{/-}$or $\mathrm{Shp}^{-/ /}$mice were backcrossed with C57BL/6 mice up to 10 generations to generate congenic C57BL/ $6 \mathrm{Fxr} /-$ (10) or Shp $\mathrm{F}^{-/-}$mice (11). Congenic single-knockout animals were bred with each other to generate FXR/SHP double-knockout mice. Age-matched, male, DKO congenic and C57BL/ 6 WT mice, 3 to 12 weeks old, were used throughout this study. WT control mice (C57BL/6) were purchased from Harlan Sprague Dawley Inc. Mice were sacrificed at 3, 5, or 10-12 weeks after birth. For the progesterone experiment, mice were injected i.p. with $125 \mathrm{mg} / \mathrm{kg}$ 17-OHP (SigmaAldrich) or corn oil twice on the first day, followed by once daily injections for 4 additional days. Blood was collected and liver tissue was frozen in liquid nitrogen. Mice were housed on a standard 12-hour-light/dark cycle and were fed normal chow and water ad libitum. All animal experiments were carried out as outlined in the Guide for the Care and Use of Laboratory Animals, prepared by the National Academy of Sciences and published by the NIH (NIH publication 86-23, revised 1985). Animal experiments were approved by the Institutional Animal Care and Use Committee at Baylor College of Medicine (protocol AN-4746 and AN-1550).

Histology. Liver samples were fixed in $10 \%$ formalin or $2.5 \%$ glutaraldehyde solution or snap frozen in OCT. The formalin fixed sections were used for H\&E staining as well as immunohistochemical detection of Ki-67 and CK19. Briefly, the sections were incubated with anti-Ki-67 antibody (Novus Biologicals) at 1:200, followed by DAB staining and hematoxylin counter-staining. For CK19 immunostaining, the rabbit antibody was provided by X. Wang, University of Michigan, Ann Arbor, Michigan, USA, and was used at 1:600 dilution. On the other hand, frozen sections were incubated with oil red $\mathrm{O}$, washed with $70 \%$ ethanol, and counterstained with hematoxylin.

Electron microscopy. Glutaraldehyde-fixed sections were embedded in plastic and stained with osmium tetroxide or uranyl acetate for examination in a FEI Tecnai Spirit transmission electron microscope.

Serum analysis. Blood was collected and centrifuged at $6,000 \mathrm{~g}$ for $5 \mathrm{~min}-$ utes to separate serum. Serum biochemistry was performed in the Comparative Pathology Laboratory at Baylor College of Medicine. Total serum BAs were measured with a BA L3K assay kit (Diagnostic Chemicals). Then, $2-5 \mu \mathrm{l}$ of serum was incubated with the given reagents for 10 minutes at $37^{\circ} \mathrm{C}$ and read at $405 \mathrm{~nm}$ with $660 \mathrm{~nm}$ as reference. Serum 17-OHP and DHEA were measured using kits from Beckman Coulter Inc.

Tissue BA analysis. One hundred to one hundred and fifty $\mathrm{mg}$ of liver tissue was weighed and homogenized in $1 \mathrm{ml}$ of $75 \%$ ethanol. The homogenate was incubated at $50^{\circ} \mathrm{C}$ for 2 hours to extract BAs and centrifuged at $6,000 \mathrm{~g}$ for 10 minutes. The supernatant was used to determine the BA content, which was normalized to the weight of liver tissue used. To determine the biliary BA level, the cystic duct was tied and the intact gallbladder was removed and stored in an eppendorf tube. The gallbladder content was released into the tube by puncturing with a needle. The BA concentrations were measured using the colorimetric assay, as described above.

Analysis of hepatic and serum BA composition. Serum or livers from 6 mice of each genotype were pooled and processed for BA determination using HPLC (Shimadzu), as described previously (70).
Gene expression analysis. Total RNA from liver was prepared according to the manufacturer's protocols (TRIzOL, Invitrogen). The RNA obtained was further purified using Qiagen columns for microarray and NanoString analysis.

Microarray analysis. Five hundred ng of total RNA was reverse transcribed into cRNA and biotin-UTP labeled using the Illumina TotalPrep RNA Amplification Kit (Ambion). cRNA was quantified using an Agilent Bioanalyzer 2100 and hybridized to the Illumina MouseRefseq-8 Expression BeadChip using standard protocols (Illumina). Image data were processed using Illumina BeadStudio software. Statistical analysis of differential gene expression was analyzed using R and Bioconductor (BioC) (71). Briefly, the unnormalized probe values from BeadStudio were read into R using the lumi BioC (72) package, variance stabilized (73), and quantile normalized. Normalized array features were filtered for probes with present calls $>0$ prior to differential gene expression analysis. The limma BioC package (74) was used to determine differentially expressed genes, using a linear model fit to a group-means parameterization. GO enrichment analysis of significant gene lists was performed using a hypergeometric test, as outlined in the GOstats BioC (75) package. The versions of software and BioC packages were as follows: R_2.8.0, lumi_1.8.2, limma_2.18.2, GOstats_2.8.0, and GO.db_2.2.5. The GEO accession number for the array data set is GSE20599.

NanoString $n$ Counter assay. The details of the nCounter Analysis System (NanoString Technologies) were reported previously (39). In brief, sequencespecific probes were constructed for each gene of interest (Supplemental Table 1). Each of the 48 samples was hybridized in duplicates with $100 \mathrm{ng}$ total RNA in each reaction. All genes and controls were assayed simultaneously in multiplexed reactions (39). The raw data was normalized in a 2-step fashion. First, it was normalized to the spike control used in each reaction to account for variations in hybridization and purification efficiency. Then, the data was normalized to $\beta$-actin, a housekeeping gene.

Western blot analysis. Protein was extracted from $100 \mathrm{mg}$ fresh liver with a Dounce homogenizer in $10 \mathrm{mM}$ HEPES ( $\mathrm{pH} 7.5$ ), $0.32 \mathrm{M}$ sucrose, $1 \%$ SDS, $5 \mu \mathrm{M}$ MG132, and $5 \mathrm{mM}$ EDTA with protease inhibitors. For Western blot, $30-50 \mu \mathrm{g}$ total protein was used. The membrane was incubated with a Cyp7a1 (obtained from David Russell) or Cyp17a1 antibody (Santa Cruz Biotechnology Inc.) at a dilution of 1:1,000.

Statistics. Data was analyzed using 1-way ANOVA, with post-hoc Bonferroni test for comparison of multiple groups or unpaired Student's $t$ test for comparison between 2 groups. $P$ values of less than 0.05 were considered significant.

\section{Acknowledgments}

The authors would like to thank the NIDDK, Digestive Disease Center Morphology Core (grant DK56338), Houston, Texas, USA, and especially Jim Barrish for help with electron microscopy and Angela Major for help with immunohistochemistry. We would like to thank David Russell for providing us with the Cyp7a1 antibody. We would also like to thank the University of Virginia Center for Research in Reproduction Ligand Assay and Analysis Core, supported by the Eunice Kennedy Shriver NICHD/NIH (SCCPIR) grant U54-HD28934, for 17-OHP and DHEA analysis. This study was supported by NIDDK grants DK068804 (to D.D. Moore) and DK62434 (to D.D. Moore and N.J. McKenna) and the R.P. DohertyJr. Welch Chair in Science (to D.D. Moore).

Received for publication August 13, 2010, and accepted in revised form October 13, 2010.

Address correspondence to: David D. Moore, 1 Baylor Plaza, N610 Alkek Building, Baylor College of Medicine, Houston, Texas 77030, USA. Phone: 713.798.3313; Fax: 713.798.3017; E-mail: moore@bcm.edu. 
1. Hofmann AF. Bile acids, cholesterol, gallstone calcification, and the enterohepatic circulation of bilirubin. Gastroenterology. 1999;116(5):1276-1277.

2. Huang W, et al. Nuclear receptor-dependent bile acid signaling is required for normal liver regeneration. Science. 2006;312(5771):233-236

3. Houten SM, Watanabe M, Auwerx J. Endocrine functions of bile acids. EMBO J. 2006;25(7):1419-1425.

4. Watanabe $M$, et al. Bile acids induce energy expenditure by promoting intracellular thyroid hormone activation. Nature. 2006;439(7075):484-489.

5. Watanabe $\mathrm{M}$, et al. Bile acids lower triglyceride levels via a pathway involving FXR, SHP, and SREBP1c. J Clin Invest. 2004;113(10):1408-1418.

6. Thomas C, et al. TGR5-mediated bile acid sensing controls glucose homeostasis. Cell Metab. 2009;10(3):167-177.

7. Goodwin B, et al. A regulatory cascade of the nuclear receptors FXR, SHP-1, and LRH-1 represses bile acid biosynthesis. Mol Cell. 2000;6(3):517-526.

8. Makishima $\mathrm{M}$, et al. Identification of a nuclear receptor for bile acids. Science. 1999;284(5418):1362-1365.

9. Parks DJ, et al. Bile acids: natural ligands for an orphan nuclear receptor. Science. 1999;284(5418):1365-1368.

10. Sinal CJ, Tohkin M, Miyata M, Ward JM, Lambert G, Gonzalez FJ. Targeted disruption of the nuclear receptor FXR/BAR impairs bile acid and lipid homeostasis. Cell. 2000;102(6):731-744.

11. Wang $\mathrm{L}$, et al. Redundant pathways for negative feedback regulation of bile acid production. Dev Cell. 2002;2(6):721-731.

12. Lee HK, et al. Structure and expression of the orphan nuclear receptor SHP gene. J Biol Chem. 1998; 273(23):14398-14402.

13. Kerr TA, et al. Loss of nuclear receptor SHP impairs but does not eliminate negative feedback regulation of bile acid synthesis. Dev Cell. 2002;2(6):713-720.

14. Jelinek DF, Andersson S, Slaughter CA, Russell DW. Cloning and regulation of cholesterol 7 alphahydroxylase, the rate-limiting enzyme in bile acid biosynthesis. J Biol Chem. 1990;265(14):8190-8197.

15. Russell DW. The enzymes, regulation, and genetics of bile acid synthesis. Annu Rev Biochem. 2003; 72:137-174.

16. Sokol RJ, Devereaux M, Dahl R, Gumpricht E. "Let there be bile"--understanding hepatic injury in cholestasis. J Pediatr Gastroenterol Nutr. 2006; 43(suppl 1):S4-S9.

17. Suchy FJ, Sokol R, Balistreri WF, eds. Liver Disease In Children. 3rd ed. New York, New York, USA: Cambridge University Press; 2007.

18. Suchy FJ. Neonatal cholestasis. Pediatr Rev. 2004; 25(11):388-396.

19. Rodriguez-Garay EA. Cholestasis: human disease and experimental animal models. Ann Hepatol. 2003;2(4):150-158.

20. Huang L, et al. Farnesoid X receptor activates transcription of the phospholipid pump MDR3. J Biol Chem. 2003;278(51):51085-51090.

21. Ananthanarayanan M, Balasubramanian N, Makishima M, Mangelsdorf DJ, Suchy FJ. Human bile salt export pump promoter is transactivated by the farnesoid X receptor/bile acid receptor. J Biol Chem. 2001;276(31):28857-28865

22. Denson LA, et al. The orphan nuclear receptor, shp, mediates bile acid-induced inhibition of the rat bile acid transporter, ntcp. Gastroenterology. 2001;121(1):140-147

23. Thomas C, Pellicciari R, Pruzanski M, Auwerx J, Schoonjans K. Targeting bile-acid signalling for metabolic diseases. Nat Rev Drug Discov. 2008;7(8):678-693.

24. Lu TT, et al. Molecular basis for feedback regulation of bile acid synthesis by nuclear receptors. Mol Cell. 2000;6(3):507-515

25. Hwang ST, Urizar NL, Moore DD, Henning SJ. Bile acids regulate the ontogenic expression of ileal bile acid binding protein in the rat via the farnesoid $\mathrm{X}$ receptor. Gastroenterology. 2002;122(5):1483-1492.

26. Landrier JF, Eloranta JJ, Vavricka SR, Kullak-Ublick GA. The nuclear receptor for bile acids, FXR, transactivates human organic solute transporter-alpha and -beta genes. Am J Physiol Gastrointest Liver Physiol. 2006;290(3):G476-G485.

27. Inagaki $\mathrm{T}$, et al. Fibroblast growth factor 15 functions as an enterohepatic signal to regulate bile acid homeostasis. Cell Metab. 2005;2(4):217-225.

28. Song KH, Li T, Owsley E, Strom S, Chiang JY. Bile acids activate fibroblast growth factor 19 signaling in human hepatocytes to inhibit cholesterol 7alpha-hydroxylase gene expression. Hepatology. 2009;49(1):297-305.

29. Guo GL, et al. Complementary roles of farnesoid $\mathrm{X}$ receptor, pregnane $\mathrm{X}$ receptor, and constitutive androstane receptor in protection against bile acid toxicity. J Biol Chem. 2003;278(46):45062-45071.

30. Seol W, Choi HS, Moore DD. An orphan nuclear hormone receptor that lacks a DNA binding domain and heterodimerizes with other receptors. Science. 1996;272(5266):1336-1339.

31. Lee YK, Dell H, Dowhan DH, Hadzopoulou-Cladaras $\mathrm{M}$, Moore DD. The orphan nuclear receptor SHP inhibits hepatocyte nuclear factor 4 and retinoid X receptor transactivation: two mechanisms for repression. Mol Cell Biol. 2000;20(1):187-195.

32. Lee YK, Moore DD. Dual mechanisms for repression of the monomeric orphan receptor liver receptor homologous protein- 1 by the orphan small heterodimer partner. J Biol Chem. 2002;277(4):2463-2467.

33. Boulias K, Katrakili N, Bamberg K, Underhill P, Greenfield A, Talianidis I. Regulation of hepatic metabolic pathways by the orphan nuclear receptor SHP. Embo J. 2005;24(14):2624-2633

34. Wang L, Han Y, Kim CS, Lee YK, Moore DD. Resistance of SHP-null mice to bile acid-induced liver damage. J Biol Chem. 2003;278(45):44475-44481.

35. Li G, Thomas AM, Hart SN, Zhong X, Wu D, Guo GL. Farnesoid $X$ receptor activation mediates head-to-tail chromatin looping in the $\mathrm{Nr} 0 \mathrm{~b} 2$ gene encoding small heterodimer partner. Mol Endocrinol. 2010;24(7):1404-1412.

36. Hartgens F, Kuipers H. Effects of androgenic-anabolic steroids in athletes. Sports Med. 2004;34(8):513-554.

37. Pavlatos AM, Fultz O, Monberg MJ, Vootkur A, Pharmd. Review of oxymetholone: a 17alphaalkylated anabolic-androgenic steroid. Clin Ther. 2001;23(6):789-801; discussion 771.

38. Welder AA, Robertson JW, Melchert RB. Toxic effects of anabolic-androgenic steroids in primary rat hepatic cell cultures. J Pharmacol Toxicol Methods. 1995;33(4):187-195.

39. Geiss GK, et al. Direct multiplexed measurement of gene expression with color-coded probe pairs. Nat Biotechnol. 2008;26(3):317-325.

40. Blake JA, Harris MA. The Gene Ontology (GO) project: structured vocabularies for molecular biology and their application to genome and expression analysis. Curr Protoc Bioinformatics. 2008; Chapter 7:Unit 7.2

41. Barrell D, Dimmer E, Huntley RP, Binns D, O'Donovan C, Apweiler R. The GOA database in 2009--an integrated Gene Ontology Annotation resource. Nucleic Acids Res. 2009;37(Database issue):D396-D403.

42. Miller WL. Steroidogenic enzymes. Endocr Dev. 2008;13:1-18.

43. Miller WL, Auchus RJ, Geller DH. The regulation of 17,20 lyase activity. Steroids. 1997;62(1):133-142.

44. Miller WL, Geller DH, Auchus RJ. The molecular basis of isolated 17,20 lyase deficiency. Endocr Res. 1998;24(3-4):817-825.

45. Miller WL. Androgen synthesis in adrenarche. Rev Endocr Metab Disord. 2009;10(1):3-17.

46. Olgemoller B, Roscher AA, Liebl B, Fingerhut R. Screening for congenital adrenal hyperplasia: adjustment of 17-hydroxyprogesterone cut-off values to both age and birth weight markedly improves the predictive value. $J$ Clin Endocrinol Metab. 2003;88(12):5790-5794.

47. Wagner M, Zollner G, Trauner M. New molecular insights into the mechanisms of cholestasis. JHepatol. 2009;51(3):565-580.

48. Chen F, et al. Progressive familial intrahepatic cholestasis, type 1 , is associated with decreased farnesoid X receptor activity. Gastroenterology. 2004;126(3):756-764.

49. Alvarez L, et al. Reduced hepatic expression of farnesoid X receptor in hereditary cholestasis associated to mutation in ATP8B1. Hum Mol Genet. 2004;13(20):2451-2460

50. Marzolini C, et al. A common polymorphism in the bile acid receptor farnesoid $\mathrm{X}$ receptor is associated with decreased hepatic target gene expression. $\mathrm{Mol}$ Endocrinol. 2007;21(8):1769-1780.

51. Van Mil SW, et al. Functional variants of the central bile acid sensor FXR identified in intrahepatic cholestasis of pregnancy. Gastroenterology. 2007;133(2):507-516

52. Demeilliers C, et al. Altered hepatobiliary gene expressions in PFIC1: ATP8B1 gene defect is associated with CFTR downregulation. Hepatology. 2006;43(5):1125-1134.

53. Zhang Y, Klaassen CD. Effects of feeding bile acids and a bile acid sequestrant on hepatic bile acid composition in mice. J Lipid Res. 2010;51(11):3230-3242.

54. Kurbegov AC, et al. Biliary diversion for progressive familial intrahepatic cholestasis: improved liver morphology and bile acid profile. Gastroenterology. 2003;125(4):1227-1234.

55. Emerick KM, et al. Bile composition in Alagille Syndrome and PFIC patients having Partial External Biliary Diversion. BMC Gastroenterol. 2008;8:47.

56. Erker L, Grompe M. Signaling networks in hepatic oval cell activation. Stem Cell Res. 2007;1(2):90-102.

57. Schuetz EG, et al. Disrupted bile acid homeostasis reveals an unexpected interaction among nuclear hormone receptors, transporters, and cytochrome P450. J Biol Chem. 2001;276(42):39411-39418.

58. Sokol RJ, Winklhofer-Roob BM, Devereaux MW, McKim JM Jr. Generation of hydroperoxides in isolated rat hepatocytes and hepatic mitochondria exposed to hydrophobic bile acids. Gastroenterology. 1995;109(4):1249-1256.

59. Grasfeder LL, et al. Fasting-induced hepatic production of DHEA is regulated by PGC-1alpha, ERRalpha, and HNF4alpha. Mol Endocrinol. 2009;23(8):1171-1182.

60. Liu Y, Yao ZX, Papadopoulos V. Cytochrome P450 17alpha hydroxylase/17,20 lyase (CYP17) function in cholesterol biosynthesis: identification of squalene monooxygenase (epoxidase) activity associated with CYP17 in Leydig cells. Mol Endocrinol. 2005;19(7):1918-1931.

61. Nakamura Y, Xing Y, Sasano H, Rainey WE. The mediator complex subunit 1 enhances transcription of genes needed for adrenal androgen production. Endocrinology. 2009;150(9):4145-4153.

62. Attardi BJ, Zeleznik A, Simhan H, Chiao JP, Mattison DR, Caritis SN. Comparison of progesterone and glucocorticoid receptor binding and stimulation of gene expression by progesterone, 17-alpha hydroxyprogesterone caproate, and related progestins. Am J Obstet Gynecol. 2007; 197(6):599.e1-599.e7.

63. Migeon CJ, Wisniewski AB. Congenital adrenal hyperplasia owing to 21-hydroxylase deficiency. Growth, development, and therapeutic considerations. Endocrinol Metab Clin North Am. 2001;30(1):193-206.

64. Falhammar $\mathrm{H}$, et al. Increased liver enzymes in adult women with congenital adrenal hyperplasia due to 21-hydroxylase deficiency. Endocr J. 2009;56(4):601-608.

65. Yamamoto Y, et al. Estrogen receptor alpha mediates 17alpha-ethynylestradiol causing hepatotoxicity. 
J Biol Chem. 2006;281(24):16625-16631.

66. MacDonald BS, Sykes PJ, Kilshaw BH, Harkness RA. Proceedings: Metabolism of the hydroxymethylene group in the anabolic steroid, oxymetholone: isolation and identification of two major metabolites. J Endocrinol. 1973;59(2):18-19.

67. Brown H, Willardson DG, Samuels LT, Tyler FH. 17-Hydroxycorticosteroid metabolism in liver disease. J Clin Invest. 1954;33(11):1524-1532.

68. Bacq Y, Sapey T, Brechot MC, Pierre F, Fignon A, Dubois F. Intrahepatic cholestasis of pregnancy: a French prospective study. Hepatology. 1997;26(2):358-364.
69. Glantz A, Reilly SJ, Benthin L, Lammert F, Mattsson LA, Marschall HU. Intrahepatic cholestasis of pregnancy: Amelioration of pruritus by UDCA is associated with decreased progesterone disulphates in urine. Hepatology. 2008;47(2):544-551.

70. Sakakura H, Suzuki M, Kimura N, Takeda H, Nagata S, Maeda M. Simultaneous determination of bile acids in rat bile and serum by high-performance liquid chromatography. J Chromatogr. 1993;621(2):123-131.

71. Gentleman RC, et al. Bioconductor: open software development for computational biology and bioinformatics. Genome Biol. 2004;5(10):R80.
72. Du P, Kibbe WA, Lin SM. lumi: a pipeline for processing Illumina microarray. Bioinformatics. 2008;24(13):1547-1548

73. Lin SM, Du P, Huber W, Kibbe WA. Model-based variance-stabilizing transformation for Illumina microarray data. Nucleic Acids Res. 2008;36(2):e11.

74. Smyth GK. Linear models and empirical bayes methods for assessing differential expression in microarray experiments. Stat Appl Genet Mol Biol. 2004;3:Article3.

75. Falcon S, Gentleman R. Using GOstats to test gene lists for GO term association. Bioinformatics. 2007;23(2):257-258. 